\title{
Protein expression of MEF2C during the critical period for visual development in vervet monkeys
}

\author{
Daniel M Bernad*, Pascal E Lachance, Avijit Chaudhuri
}

\begin{abstract}
During the early development of the visual cortex, there is a critical period when neuronal connections are highly sensitive to changes in visual input. Deprivation of visual stimuli during the critical period elicits robust anatomical and physiological rearrangements in the monkey visual cortex and serves as an excellent model for activity-dependent neuroplasticity. DNA microarray experiments were previously performed in our lab to analyze gene expression patterns in area $\mathrm{V} 1$ of vervet monkeys subjected to monocular deprivation (MD). An interesting candidate identified in its screen was myocyte enhancer-binding factor 2C (MEF2C), a transcription factor linked to neuronal survival. Consistent with the microarray data, we show that there is a qualitative increase in MEF2C protein expression in area V1 of infant as compared to adult vervet monkeys. Our results suggest that the regulation of neuronal survival is one of the molecular mechanisms underlying the critical period for visual cortical neuroplasticity.
\end{abstract}

KEYWORDS: MEF2C, Neural plasticity, ocular dominance columns, visual cortical development, critical period, CaMK cell-signaling cascade

\section{INTRODUCTION}

A set of classic experiments performed by Hubel and Wiesel (1) demonstrated that intercellular communication between neurons is morphologically and physiologically plastic in response to deprivation of visual stimuli. The authors found that the visual cortex is arranged in ocular dominance columns (ODCs), where alternating columns of neurons preferentially respond to visual stimuli presented to either eye $(1,2)$. Also, electrophysiological recording demonstrate that deprivation of visual stimuli to one eye leads to a reduction in neuronal activity in the ODCs that respond to that eye. Furthermore, in infants subjected to monocular deprivation (MD), anatomical rearrangement of ODCs occur such that cortical areas driven by the open eye increase in size at the expense of those initially responding to the deprived eye. However, this process was not observed in adults subjected to $\mathrm{MD}$, indicating that there is a "critical period" during the early development when the brain is capable of

*To whom correspondence should be addressed:

Daniel M Bernad

320 Freedom Private, Ottawa, Ontario, Canada, K1G6W4

Email: danbernad@gmail.com adjusting connections in response to changes in the visual environments (3).

The monkey visual system is anatomically and functionally similar to that of human (4). In primates, the plastic nature of the visual cortex in response to external stimuli during the critical period serves as a robust model for cortical neuroplasticity $(5,6)$. Thus, deprivation of visual stimuli can therefore be used as an in vivo model for the study of the molecular mechanisms underling these neuroplastic responses. Previous work employing Microarray analysis identified a novel candidate, myocyte enhancer-binding factor $2 \mathrm{C}(\mathrm{MEF} 2 \mathrm{C})$, as a potential regulator of development (7). MEF2C is expressed in a variety of tissues, among which its function in the development of cardiac muscle cells has been well described $(8,9)$. Lachance and Chaudhuri (7) showed MEF2C mRNA expression was increased roughly 2-fold in infants compared to adults. In addition, a trend suggested that the levels of MEF2C mRNA may be further increased by MD (10). This raises the possibility that changes in MEF2C activity are important molecular mechanisms underlying the rearrangements that can occur during the critical period.

Generally, cell-signaling pathways involve 
intracellular protein-protein interactions. In particular, the calcium/calmodulin dependent kinase pathway has been linked to neuroplasticity (11). Upon binding calcium, calmodulin $(\mathrm{CaM})$ activates several proteins including calmodulin-dependent protein kinases (CaMKs). The initiation of these signaling pathways eventually leads to the downstream activation of MEF2C which promotes neuronal cell survival and cortical differentiation (12). The present study will examine MEF2C expression employing visual cortical neuroplasticity as a model system.

In this study, Western blot analyses were performed to validate the MEF2C microarray results at the protein level. Although rates of protein expression often follow changes in mRNA transcription, in numerous cases, gene expression is also regulated at the level of translation (4). It is therefore important to verify that the protein expression profile is similar to that of the mRNA. We hypothesized that MEF2C protein expression is higher in area $\mathrm{V} 1$ of infants in comparison to adults, consistent with the microarray expression data. In addition, we investigated whether MEF2C expression is modulated by monocular enucleation (ME), as suggested by a trend observed in the GeneChip ${ }^{\circledR}$ analysis.

\section{MATERIALS \& METHODS}

\section{Animals and tissue preparation}

For this experiment, frozen samples from twelve individual vervet monkeys (Cercopoithecus aethiops) were obtained from a bank of brain tissue. The brain tissues used were from the same animals examined in the microarray analysis performed by Lachance \& Chaudhuri (10). As previously described (13), brain tissue was acquired from infant and adult vervet monkeys from our feral population in St. Kitts, West Indies. Briefly, adult and infant (27-35 days of age) monkeys were subjected to ME followed by survival of 1 or 5 days. Control animals were raised with binocular vision. The monkeys were initially sedated with ketamine $(10 \mathrm{mg} / \mathrm{kg}$, i.m.) and the euthanized via an overdose of sodium pentobarbital $(2 \mathrm{mg} / \mathrm{kg}$, i.v.). Animals were then perfused transcardially with DEPCtreated phosphate buffered saline (PBS, $2.03 \mathrm{~g}$ $\mathrm{NaH} 2 \mathrm{PO} 4, \quad 11.49 \mathrm{~g} \quad \mathrm{Na} 2 \mathrm{HPO} 4, \quad 85 \mathrm{~g} \quad \mathrm{NaCl})$. Subsequently, the primary visual cortex (area V1) was removed from the excised brain by blocking along the lunate sulcus and was frozen in liquid nitrogen/isopenate. The tissue specimen was equilibrated in dry ice, transported to Montreal, and stored in a $-80^{\circ} \mathrm{C}$ freezer (14). All experiments were conducted in accordance of standard guidelines of the Canadian Council for Animal Care and were peerreviewed by an institutional Animal Care Committee.

\section{Western blot analysis}

Protein extracts were prepared on ice, from frozen fragments (approx. 2000 $\mu \mathrm{g}$ ) of visual cortex tissue (area V1), by homogenization in RIPA Buffer $(50 \mathrm{mM}$ Tris$\mathrm{HCl} \mathrm{pH} 8.0,300 \mathrm{mN} \mathrm{NaCl}, 0.5 \%$ Nonidet P-40, $0.5 \%$ deoxycholate, $0.1 \%$ SFS) supplemented with Complete Protease Inhibitor Cocktail, was quantified, and subsequently stored as described by Lalonde et al. (15). Gel electrophoresis was performed as described by Lalonde et al. (15). Proteins were then transferred to a Hybond-C Extra nitrocellulose membrane (Amersham, Peapack, NJ) using the Novex XCell II Blot Module at $20 \mathrm{~mA}$ for 2 hours. Confirmation of transfer was assessed by Ponceau S staining. The membrane was blocked in $2 \%$ Blotto $(2 \%(\mathrm{w} / \mathrm{v})$ carnation non-fat powdered milk, 0.1 M Tris-HCl pH 7.5, $0.15 \mathrm{M} \mathrm{NaCl}$, $0.1 \%$ Tween-20) for 1 hour. The blot was then incubated overnight at $4^{\circ} \mathrm{C}$ with primary antisera in a sealed plastic bag, washed four times with 2\% Blotto for 15 min. Blots were then incubated with Horse Radish Peroxidase (HRP)-conjugated secondary antibody at (1:5000) and washed four times with $2 \%$ Blotto for 5 min. Chemiluminescence and visualization was performed as described by Lalonde et al. (15).

\section{Antisera}

Antisera were obtained from commercial sources. The polyclonal MEF2C antisera was raised in rabbits against a KLH-coupled synthetic peptide based on the sequence surrounding serine- 419 of human MEF2C and purified by protein $\mathrm{A}$ and peptide affinity chromatography (New England Biolabs, Ipswich, Ma). The monoclonal serum against GAPDH (glyceraldehydes-3-phosphatedehydrogenase) was raised in mouse cells against GAPDH purified from rabbit muscles (Chemicon International, Temecula, $\mathrm{Ca}$ ). HRP-conjugated secondary antisera against mouse IgG were obtained from Amersham (Peapack, NJ). GAPDH titration was performed on the same Western blot probed with MEF2C.

\section{RESULTS}

To demonstrate that MEF2C protein expression is more elevated in area V1 of infants, we examined its expression in protein extracts harvested from brain tissue of two individual monkeys of each age group (Fig. 1). Our results show that MEF2C expression is qualitatively higher in infants than adults. Furthermore, we observed a single $45 \mathrm{kDa}$ band which was reproducible in the two individual monkeys tested. Incubation with the $\alpha \mathrm{GAPDH}$ control confirms that similar amounts of protein extract was loaded in each lane (Fig. 1). Microarray data also raised the possibility that MEF2C expression in the visual cortex is activity- 


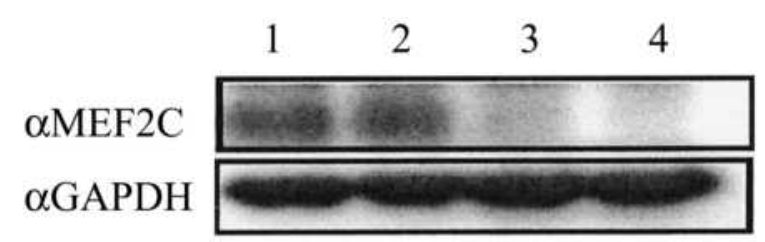

Figure 1: MEF2C protein expression is higher in area V1 of infant compared to adult monkeys. Protein extract from the visual cortex of infants (lanes 1 and 2) and adults (lanes 3 and 4) was assayed with $\alpha$ MEF2C (1:1000 dilution). Incubation of the same blot with $\alpha \mathrm{GAPDH}$ (1:50 dilution) controls that similar amounts of protein were leaded in each lane.

dependent during that critical period (10). To assess whether MEF2C protein expression is modulated by activity, the levels of MEF2C were examined in extracts prepared from normal infants and adults and were compared to animals subjected to $\mathrm{ME}$ followed by 1 to 5 day survival (Fig. 2). Although showing a consistently higher expression of MEF2C in all three infant experimental conditions, we did not observe an increase of $\mathrm{MEF} 2 \mathrm{C}$ as a result of ME. Our results suggest that MEF2C is expressed at higher levels in area V1 of infant as compared to adult vervet monkeys, and is not modulated in an activity-dependent manner.

\section{DISCUSSION}

Based on our results from a microarray screen that identified RNA expression patterns which correlated with neuroplastic rearrangements during the critical period of visual cortical development (10), we examined the protein expression profile of the transcription factor MEF2C. To this end, we characterized the cross-species reactivity of antisera generated against MEF2C and GAPDH to use as tools to study their expression in extracts of area $\mathrm{V} 1$ of vervet monkeys. Our results show that MEF2C is expressed at higher levels in area V1 of infant as compared to adult monkeys.

Previous work suggests that the establishment of ODCs occur early during infancy (pre-natal in monkeys) and is independent of the critical period, which peaks at 25-35 days of age in monkeys (16). The increase in MEF2C expression in 27-35 day old infants suggests it may have a role in the molecular events that underlie the critical period. However, to better characterize the correlation between MEF2C expression and the critical period, a more defined time-course of MEF2C expression, one that includes time points before and after the critical period in normal infants, should be performed.

Our results also suggest that $\mathrm{MEF} 2 \mathrm{C}$ protein expression in area V1 is not modulated by MD. In corroboration of this finding, Leysen et al. (17) failed to show statistically significant differences in MEF2C protein expression in normal adult cats and those

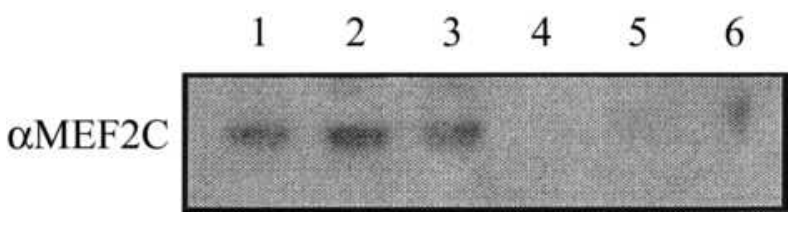

Figure 2: MEF2C protein expression is not modulated in correlation with monocular deprivation. A Western assayed with $\alpha$ MEF2C (1:1000 dilution) was used to compare the levels of MEF2C in area V1 of normal animals (lane 1, infant A; lane 4, adult A) with animals subjected to MD followed by 1 day (lane 2 , infant $\mathrm{B}$; lane 15 , adult $\mathrm{B}$ ) or 5 days of survival (lane 3 , infant $C$; lane 6 , adult $C$ ).

subjected to retinal lesions. In addition, Microarray data suggested a non-significant correlation between elevation of MEF2C mRNA expression and MD (10). One possibility is that in response to $\mathrm{MD}$, the expression of MEF2C mRNA is modulated differently than its translation into protein. However, the trend showing that MEF2C mRNA levels increase in correlation with $\mathrm{MD}$ was not replicated in a Northern analysis of MEF2C expression in the same animals (Lachance and Chaudhuri, personal communication). Taken together, these results suggest that the expression of both MEF2C mRNA and protein in the visual cortex is not activity-dependent.

Although MEF2C levels do not change in correlation with $\mathrm{MD}$, it is possible that MEF2C activity is modulated post-translationally in a manner that would correlate with neuroplastic rearrangements in area V1 during the critical period. It has been well characterized that $\mathrm{MEF} 2 \mathrm{C}$ activity is positively regulated subsequent to phosphorylation by $\mathrm{p} 38$ MAPK and CaMKIV (12).The p38 MAPK and CaMKIV pathways are induced in cells undergoing neuroplastic changes and promote neuronal survival when activating MEF2C during development (12). However, in mature neurons exposed to excitotoxic stresses, the MAPK pathway is pro-apoptotic because it leads to the activation of caspases which cleave MEF2C proteins, including MEF2C (18). It is possible that while the levels of MEF2C remain unaffected, the phosphorylation status of MEF2C may change in correlation with ME in area V1 during the critical period of visual cortical development. This hypothesis could be tested when an antiserum specific for the phosphorylated forms of MEF2C becomes available.

The regulators of MEF2C activity, CaM type 1 and CaMKIV, have also been identified in the microarray screen for genes whose expression is modulated during the critical period of visual cortical neuroplasticity (10). CaM type 1 expression appears elevated in infants in comparison to adults, whereas CaMKIV seems to be induced by ME during the critical period. Future work should focus on the expression of the protein products for these genes to further validate the microarray data. 
In addition, the protein expression patterns of other regulators of MEF2C activity, such as P38MAPK, should be examined in similar experimental conditions. Increase in expression of several proteins in the pathway leading to changes in MEF2C activity suggests that the regulation of neuronal survival is one of the molecular mechanisms underlying the critical period for visual cortical neuroplasticity. Directly showing the importance of MEF2C during the critical period of development via gain and loss of function genetic experiments would be helpful in elucidating its definitive role in the neuroplastic response of the visual cortex.

\section{CONCLUSION}

This study has demonstrated a qualitative increase in $\mathrm{MEF} 2 \mathrm{C}$ protein expression in area $\mathrm{V} 1$ of infant as compared to adult vervet monkeys and further suggests that expression is not mediated in an activity-driven manner. Our results suggest that the regulation of neuronal survival is one of the molecular mechanisms underlying the critical period for visual cortical neuroplasticity.

\section{ACKNOWLEDGMENTS}

The authors would like to thank Lauren Kosowan for her formatting assistance, Cary Kogan for his editing suggestions, Shahin Zangenehpour for his stylistic assistance, and Carmelo Milo for his technical support. This study was funded by a genomics program grant from the Natural Sciences and Engineering Research Council of Canada (NSERC) awarded to AC and undergraduate student research award from NSERC to DB.

\section{REFERENCES}

1. Hubel DH, Wiesel TN. Receptive fields, binocular interaction and functional architecture in the cat's visual cortex. J. Physiol. (London) 1962 Jan;160:106-54.

2. Katz L. What's critical for the critical period of visual cortex? Cell. 1999 Dec 23;99(7):673-6.

3. Antonini A, Stryker MP Rapid remodeling of axonal arbors in the visual cortex. Science. 1993 Jun 18;260(5115):1819-21.
4. Kandel ER, Schwartz JH, Jessel TM. Central Visual Processing. In: Wurtz RH, Kandel ER,editors. Principles of Neural Science. New York: McGraw Hill; 2000. p. 521-545.

5. Gordon JA. Cellular mechanisms of visual cortical plasticity: a game of cat and mouse. Learn Mem. 1997 Sep-Oct;4(3):245-61.

6. Toldi J, Feher O, Wolff J. Neuronal plasticity induced by neonatal monocular (and binocular) enucleation. Prog Neurobiol. 1996 Feb;48(3):191-218.

7. Lachance PE, Chaudhuri A. Microarray analysis of developmental plasticity in monkey primary visual cortex.J Neurochem. 2004 Mar;88(6):1455-69.

8. Bi W, Drake CJ, Schwarz JJ. The transcription factor MEF2Cnull mouse exhibits complex vascular malformations and reduced cardiac expression of angiopoietin 1 and VEGF. Dev Biol. 1999 Jul 15;211(2):255-67.

9. Lin Q, Lu J, Yanagisawa H, et al. Requirement of the MADSbox transcription factor MEF2C for vascular development. Development. 1998 Nov;125(22):4565-74.

10. Lachance P, Chaudhuri A. Functional genomics of primate visual cortical neuroplasticity. Society for neuroscience 31st Annual meeting.2001; San Diego, Ca.

11. Soderling T CaM-Kinases: modulators of synaptic plasticity. Curr Opin Neurobiol. 2000 Jun;10(3):375-80.

12. Mellstrom B, Naranjo JR Mechanisms of calcium-dependent transcription. Curr Opin Neurobiol. 2001 Jun;11(3):312-9.

13. Kogan CS, Zangenehpour S, Chaudhuri A. Developmental profiles of SMI-32 immunoreactivity in monkey striate cortex. Brain Res Dev Brain Res. 2000 Jan 3;119(1):85-95.

14. Chaudhuri A. Neuronal activity mapping with inducible transcription factors. Neuroreport. 1997 Nov 10;8(16):v-ix.

15. Lalonde J, Lachance PE, Chaudhuri A. Monocular enucleation induces nuclear localization of calcium/calmodulin-dependent protein kinase IV in cortical interneurons of adult monkey area V1. J Neurosci. 2004 Jan 14;24(2):554-64.

16. Crowley JC, Katz LC. Early development of ocular dominance columns. Science. 2000 Nov 17;290(5495):1321-4.

17. Leysen I, Van der Gucht, Eysel UT, Huybrechts, Vandesande F, Arckens L. Time-dependent changes in the expression of the MEF2 transcription factor family during topographic map reorganization in mammalian visual cortex.Eur J Neurosci. 2004 Aug;20(3):769-80.

18. Okamoto S, Li Z, Ju C, Scholzke M, Mathews E, Cui J, Salvensen G, Bossy-Wetzel E, Lipton S (2002) Dominantinterfering forms of MEF2C generated by caspase cleavage contribute to NMDA-induced neural apoptosis. PNAS 99:39743979.

Daniel M Bernad MSc (Oxon.), MPhil (Cantab.) is a third year medical student at the University of Ottawa. $\mathrm{He}$ received his undergraduate degree in Psychology from McGill University, his MSc in Neuroscience at Oxford University (ChCh) and MPhil in Bioscience Enterprise at Cambridge University (Sid.Sussex). His multidisciplinary research interests range from employing non-invasive methods to investigate memory processing to medical device innovation. He hopes to one day integrate his research interests and career aspirations as a practicing academic neuroradiologist. 